\title{
Fatou components of entire functions of small growth
}

\author{
XINHOU HUA $\dagger$ and CHUNG-CHUN YANG $\ddagger$ \\ $\dagger$ Institute of Mathematics, Nanjing University, Nanjing 210093, P. R. China \\ (e-mail: mahua@netra.nju.edu.cn) \\ $\$$ Department of Mathematics, HKUST, Hong Kong \\ (e-mail:mayang@uxmail.ust.hk)
}

(Received 17 September 1997 and accepted in revised form 17 March 1998)

Abstract. This paper is concerned with the dynamics of transcendental entire functions. Let $f(z)$ be a transcendental entire function. We shall study the boundedness of the components of the Fatou set $F(f)$ under some restrictions on the growth of the function. This relates to a problem due to Baker in 1981.

\section{Introduction}

The iteration theory of rational functions originated with the work of Julia [18] and Fatou [15] between 1918 and 1920. Some years later in 1926 Fatou [16] studied the case of transcendental entire functions. Transcendental entire functions share many of the dynamical properties of rational functions, but there are some major differences. The main reason for this is because of the essential singularities of transcendental entire functions (cf. [13]).

In this paper $f(z)$ always stands for a transcendental entire function. The iterates $f^{n}$ are defined by

$$
f^{0}(z)=z, \quad f^{n}(z)=f\left(f^{n-1}(z)\right), \quad n \geq 1 .
$$

A point $z$ is called normal if the sequence $\left\{f^{n}\right\}$ is normal in the sense of Montel in some neighbourhood of $z$. We define

$$
F(f)=\{z \in \mathbb{C}: z \text { is a normal point }\}
$$

and

$$
J(f)=\mathbb{C} \backslash F(f) ;
$$

they are called the Fatou set and the Julia set of $f$ respectively.

According to the definition, it is easy to verify that $F$ is open (possibly empty) and $J$ is closed. 
The components of $F(f)$ are simply called Fatou components (or stable domains): they are maximal domains of normality. According to the classical Fatou-Julia theory (cf. Fatou $[\mathbf{1 5}, \mathbf{1 6}]$ and Julia $[\mathbf{1 8}]), J(f)$ and $F(f)$ are completely invariant in the sense that

$$
z \in J(f) \Longleftrightarrow f(z) \in J(f)
$$

and

$$
z \in F(f) \Longleftrightarrow f(z) \in F(f) .
$$

A further relation given in [17] is

$$
F(f)=f(F(f)) \cup\{F(f) \cap P V(f)\},
$$

where $P V(f)$ denotes the set of the Picard exceptional values of $f$. Thus, if the Fatou set $F(f)$ contains a finite Picard exceptional value of $f$, then $F(f) \neq f(F(f))$. However, for any nonlinear rational function $R(z)$, we always have $F(R)=R(F(R)$ ).

Each component $D$ of $F(f)$ is mapped by $f$ into some component $U$ of $F(f)$, and $U \backslash f(D)$ contains at most one point. Furthermore, the set $U \backslash f(D)$ is contained in the set of the asymptotic values of $f$ [9]. If each $f^{n}(D)$ belongs to a different component of $F(f)$, the component $D$ is called wandering; if this is not the case, then there are integers $m \geq 0$ and $n \geq 1$ such that $f^{m}(D) \subset D_{1}$, where $D_{1}$ is a component of $F(f)$ such that $f^{n}\left(D_{1}\right) \subset D_{1}$. In this case, we call $D$ a pre-periodic component and, in particular, $D$ is called periodic (or invariant) if $m=0$ (or $m=0$ and $n=1$ ). For periodic component $D$ of period $m$, if $f^{n m}(z) \rightarrow \infty$ for $z \in D$ as $n \rightarrow \infty$, then $D$ is called a Baker domain. One can understand the Baker domain by means of the Hadamard-three-circle theorem:

If $f$ is analytic on the annulus $\left\{z: 0 \leq r_{1} \leq|z| \leq r_{2} \leq \infty\right\}$, then for any $r: r_{1} \leq r \leq r_{2}, \log M(r, f)$ is a convex function of $\log r$, where $M(r, f)=\sup _{|z|=r}|f(z)|$.

An example of the Baker domain is the function $f(z)=z+1+e^{-z}$ (see [15]). For this function $f, f^{n}(z) \rightarrow \infty$ as $n \rightarrow \infty$ for $\operatorname{Re} z>0$.

No nonlinear rational functions have wandering domains (see Sullivan [22]), but transcendental entire functions may (cf. Baker [4, 6], Eremenko and Lyubich [14], and so on).

If $P(z)$ is a nonlinear polynomial, it is easy to see that $\infty$ is a super-attractive fixed point and $F(f)$ contains a neighbourhood of $\infty$, thus $J(f)$ is bounded; however, if $f$ is transcendental, the case is completely different. In fact, $J(f)$ is unbounded. This can be easily seen from the Picard theorem and the complete invariance of $J(f)$.

It is natural to ask whether $F(f)$ is bounded or not for transcendental entire function $f$.

To this end, recall that the order $\lambda=\lambda(f)$, the lower order $\rho=\rho(f)$ and the type $\sigma=\sigma(f)$ of $f$ are defined to be, respectively,

$$
\begin{gathered}
\lambda=\varlimsup_{r \rightarrow \infty} \frac{\log \log M(r, f)}{\log r}, \quad \rho=\underline{\lim }_{r \rightarrow \infty} \frac{\log \log M(r, f)}{\log r}, \\
\sigma=\varlimsup_{r \rightarrow \infty} \frac{\log M(r, f)}{r^{\lambda}} .
\end{gathered}
$$


A function $f$ with $\lambda(f)=\rho(f)$ is said to be of regular growth. From the theory of value distribution theory, we can see that most meromorphic functions are not of regular growth, that is, their lower order is less than the order. Moreover, there are functions with finite lower order but the order is infinite.

Baker [5] proved the following result.

THEOREM A. If the maximum modulus $M(r, f)$ of the transcendental entire function $f$ satisfies

$$
\log M(r, f)=O\left\{(\log r)^{p}\right\} \quad \text { as } r \rightarrow \infty,
$$

where $1<p<3$, then every component of $F(f)$ is bounded.

In the same paper, he proposed the following open problem.

Question 1. (Baker's Problem) Is the restriction on the growth of $f$ sharp or not?

Concerning this problem, Stallard [21] proved the following two results.

THEOREM B. If the maximum modulus $M(r, f)$ of the transcendental entire function $f$ satisfies

$$
\log \log M(r, f)<\frac{(\log r)^{1 / 2}}{(\log \log r)^{\epsilon}} \quad(0<\epsilon<1),
$$

then every component of $F(f)$ is bounded.

THEOREM C. If the order $\lambda$ of the transcendental entire function $f$ is less than $1 / 2$ and

$$
\frac{\log M(2 r, f)}{\log M(r, f)} \rightarrow c \neq \infty
$$

as $r \rightarrow \infty$, then every component of $F(f)$ is bounded.

In this paper, we shall give some further results on Baker's problem.

Definition 1. Suppose that $C_{1}$ and $C_{2}$ are constants satisfying $C_{1}>1, C_{2}>1$. A positive number $r$ is said to be normal $\left(C_{1}, C_{2}\right)$ for $f(z)$ if

$$
\log M\left(C_{1} r, f\right)<C_{2} \log M(r, f) .
$$

We denote by $N\left(C_{1}, C_{2}\right)$ the set of numbers $r$ which are normal $\left(C_{1}, C_{2}\right)$ for $f(z)$.

Remark. From (3) we see that, for any small $\varepsilon>0$, there exists a positive number $r_{0}$ such that $\left[r_{0}, \infty\right) \subset N(2, c+\varepsilon)$.

THEOREM 1. Suppose that $f(z)$ is a transcendental entire function of order $\lambda<1 / 2$. Assume that there exist constants $C_{2}>1$ and $C_{1}>C_{2}^{2}$ such that, for sufficiently large $r_{0}$, $\left[r_{0}, \infty\right) \subset N\left(C_{1}, C_{2}\right)$. Then any component of $F(f)$ is bounded.

The following result is a supplement of Theorem 1 in some sense.

THEOREM 2. Suppose that $f(z)$ is a transcendental entire function of lower order $\rho<$ $1 / 2$. Assume that, for any $m>1$,

$$
\log M\left(r^{m}, f\right) \geq m^{2} \log M(r, f)
$$

holds for all sufficiently large $r$. Then any component of $F(f)$ is bounded. 
Remark. By the Hadamard-three-circle theorem, for any $m>1$ and any $\varepsilon>0$, one has $\log M\left(r^{m}, f\right) \geq(m-\varepsilon) \log M(r, f)$. Therefore our condition (5) is not strong.

Remark. Based on this theorem, it is reasonable to guess that Theorem 1 still holds if we replace the condition that the order is less than $1 / 2$ by the condition that the lower order is less than $1 / 2$.

Example. Take $f(z)=\sin \left(z^{1 / 2}\right) / z^{1 / 2}+z+a, a \in \mathbb{C}$. Then the lower order of $f(z)$ is $1 / 2$ and

$$
\log M(2 r, f) / \log M(r, f) \rightarrow 2^{1 / 2}
$$

as $r \rightarrow \infty$. This is stronger than our growth condition (5). Baker [5] showed that $F(f)$ has an unbounded component when $a$ is sufficiently large and positive. Thus the restriction on the lower order in the above theorems is the best possible.

Next we consider some special Fatou components. Suppose that $f(z)$ is of type 0 and order less than $1 / 2$. Bhattacharyya $[\mathbf{1 0}]$ proved that any immediate attractive domain $D$ is bounded; Baker [5] extended it to invariant components, which can be easily generalized to non-wandering components (cf. Qiao [20]). We shall prove the following result.

THEOREM 3. Suppose that $f(z)$ is a transcendental entire function with lower order $\rho=\rho(f)<1 / 2$. Then every pre-periodic component is bounded.

Example. Take $f(z)=\cos \sqrt{z+(\pi / 2)^{2}}$. Then $F(f)$ has an unbounded component $U$ which contains the origin (cf. [10]). Note that $f(0)=0$, thus $U$ is invariant. Obviously, $\rho(f)=1 / 2$. Thus the restriction on the lower order is the best possible.

\section{Some basic lemmas}

The minimum modulus of $f$ is denoted by

$$
m^{*}(r, f)=\min _{|z|=r}|f(z)| .
$$

The following result was proved by Baker [5].

LEMMA 1. Suppose that there exist sequences $R_{n}, t_{n} \rightarrow \infty$ and $c(n)>1$ such that:

(i) $\quad M\left(R_{n}, f\right)=R_{n+1}$;

(ii) $\quad R_{n} \leq t_{n} \leq R_{n}^{c(n)}$;

(iii) $m^{*}\left(t_{n}, f\right)>R_{n+1}^{c(n+1)}$ for all sufficiently large $n$.

Then all the components of $F(f)$ are bounded.

LEMMA 2. The assumptions are the same as in Theorem 1. Then there exist sequences $R_{n}, t_{n} \rightarrow \infty$ such that, for all sufficiently large $n:$

(i) $\quad M\left(R_{n}, f\right)=R_{n+1}$;

(ii) $R_{n}^{2+2 /(2 n+1)}<t_{n}<R_{n}^{2+1 / n}$,

(iii) $\log m^{*}\left(t_{n}, f\right)>\left(1-1 /(2 n+1)^{3}\right) \log M\left(R_{n}^{2+2 /(2 n+1)}, f\right)$.

Proof. Since the order of $f(z)$ is less than one, by the Weierstrass theorem we may write $f(z)$ in the form

$$
f(z)=c z^{k} g(z) \quad(c \neq 0),
$$


where

$$
g(z)=2 \prod_{n=1}^{\infty}\left(1-\frac{z}{z_{n}}\right)
$$

and $k$ is a nonnegative integer. We define

$$
G(z)=2 \prod_{n=1}^{\infty}\left(1+\frac{z}{r_{n}}\right), \quad\left(r_{n}=\left|z_{n}\right|\right) .
$$

It is easy to see that

$$
\lambda(G)=\lambda(g)=\lambda(f) .
$$

From Boas [11, p. 39, formula (3.1.4)] we have

$$
m^{*}(r, G)=|G(-r)| \leq m^{*}(r, g) \leq M(r, g) \leq M(r, G)=G(r) .
$$

Since $C_{1}>C_{2}^{2}$, we can take a constant $a \in(\lambda, 1 / 2)$ such that

$$
b:=a-\log C_{2} / \log C_{1}>0 .
$$

Let

$$
\Psi(r)=\int_{r}^{\infty} \frac{\log |1-t|-\cos (\pi a) \log (1+t)}{t^{1+a}} d t, \quad(r \geq 0) .
$$

We denote $k(a)=(1-\cos (\pi a)) / a$. Then from Kjellberg [19] (cf. Denjoy [12]) we have

$$
k(a) \frac{\log (1+r)}{r^{a}} \leq \Psi(r) \leq 10 \frac{\log (1+r)}{r^{a}} .
$$

Note that

$$
\log |G(-r)|=\sum_{n=1}^{\infty} \log \left|1-\frac{r}{r_{n}}\right|
$$

and

$$
\log |G(r)|=\sum_{n=1}^{\infty} \log \left|1+\frac{r}{r_{n}}\right|
$$

and $\sum r_{n}^{-a}$ is convergent. We thus obtain

$$
\begin{aligned}
\int_{r}^{\infty} \frac{\log G(t)}{t^{1+a}} d t & =\sum_{n=1}^{\infty} \int_{r}^{\infty} \frac{\log \left(1+t / r_{n}\right)}{t^{1+a}} d t \\
& =\sum_{n=1}^{\infty} r_{n}^{-a} \int_{r / r_{n}}^{\infty} \frac{\log (1+t)}{t^{1+a}} d t
\end{aligned}
$$

(the interchange of limiting operations being justified because everything is positive) and

$$
\begin{aligned}
\int_{r}^{\infty} \frac{\log |G(-t)|}{t^{1+a}} d t & =\sum_{n=1}^{\infty} \int_{r}^{\infty} \frac{\log \left|1-t / r_{n}\right|}{t^{1+a}} d t \\
& =\sum_{n=1}^{\infty} r_{n}^{-a} \int_{r / r_{n}}^{\infty} \frac{\log |1-t|}{t^{1+a}} d t
\end{aligned}
$$


because the integral is dominated by the convergent integral

$$
\begin{aligned}
\int_{0}^{\infty} \frac{|\log | G(-t)||}{t^{1+a}} d t & =\sum_{n=1}^{\infty} \int_{0}^{\infty} \frac{|\log | 1-t / t_{n}||}{t^{1+a}} d t \\
& =\sum_{n=1}^{\infty} r_{n}^{-a} \int_{0}^{\infty} \frac{|\log | 1-t||}{t^{1+a}} d t
\end{aligned}
$$

Therefore by (7), for any $r<R$,

$$
\begin{aligned}
\int_{r}^{R} & \frac{\log |G(-t)|-\cos (\pi a) \log G(t)}{t^{1+a}} d t \\
& =\int_{r}^{\infty}-\int_{R}^{\infty} \frac{\log |G(-t)|-\cos (\pi a) \log G(t)}{t^{1+a}} d t \\
& =\sum_{n=1}^{\infty}\left(r_{n}\right)^{-a}\left\{\Psi\left(\frac{r}{r_{n}}\right)-\Psi\left(\frac{R}{r_{n}}\right)\right\} \\
& \geq k(a) \sum_{n=1}^{\infty} \frac{\log \left(1+r / r_{n}\right)}{r^{a}}-10 \sum_{n=1}^{\infty} \frac{\log \left(1+R / r_{n}\right)}{R^{a}} \\
& =k(a) \frac{\log G(r)}{r^{a}}-10 \frac{\log G(R)}{R^{a}} .
\end{aligned}
$$

Thus, for any positive integer $n$, we have

$$
\begin{aligned}
\int_{r}^{R} & \frac{\log |G(-t)|-\left[1-1 /(2 n+1)^{3}\right] \log G(r)}{t^{1+a}} d t \\
= & \int_{r}^{R} \frac{\log |G(-t)|-\cos (\pi a) \log G(t)}{t^{1+a}} d t \\
& +\int_{r}^{R} \frac{\cos (\pi a) \log G(t)-\left[1-1 /(2 n+1)^{3}\right] \log G(r)}{t^{1+a}} d t \\
\geq & k(a) \frac{\log G(r)}{r^{a}}-10 \frac{\log G(R)}{R^{a}} \\
& +\left(-k(a)+\frac{1}{a(2 n+1)^{3}}\right)\left(\frac{1}{r^{a}}-\frac{1}{R^{a}}\right) \log G(r) \\
\geq & \frac{1}{a(2 n+1)^{3}} \frac{\log G(r)}{r^{a}}-10 \frac{\log G(R)}{R^{a}} .
\end{aligned}
$$

Since $f(z)$ is transcendental, we have

$$
\lim _{r \rightarrow \infty} \frac{\log M(r, f)}{\log r}=\infty
$$

Thus for any $K>0$, there exists $r_{1}>r_{0}>0$ such that $M(r, f)>r^{K}$ for all $r>r_{1}$. Let

$$
s_{n}=\left(10 a(2 n+1)^{3}\right)^{n(2 n+1) / b}
$$

and take

$$
R_{1} \geq \max \left\{r_{1}, 40 \log M(1, f), s_{1}\right\}
$$


We define

$$
M\left(R_{n}, f\right)=R_{n+1}, \quad n \geq 1 .
$$

Note that $\log s_{n+1} / \log s_{n} \rightarrow 1$ as $n \rightarrow \infty$. Thus when $K$ is large, we have

$$
R_{n+1}=M\left(R_{n}, f\right) \geq R_{n}^{K} \geq s_{n+1} .
$$

Define

$$
h(n)=R_{n}^{2+2 /(2 n+1)}, \quad \delta(n)=\frac{1}{4 n(n+1)} .
$$

Note that, for any $r \geq r_{1}$, by the assumptions we know that

$$
\log M\left(C_{1} r, f\right) \leq C_{2} \log M(r, f) .
$$

This implies that

$$
\log M\left(h(n)^{1+\delta(n)}, f\right) \leq C_{2}^{\delta(n) \log h(n) / \log C_{1}} \log M(h(n), f) .
$$

Applying (8) for $r=h(n)$ and $R=h(n)^{1+\delta(n)}$ we get

$$
\int_{h(n)}^{h(n)^{1+\delta(n)}} \frac{\log |G(-t)|-\left[1-1 /(2 n+1)^{3}\right] \log G(h(n))}{t^{1+a}} d t \geq \frac{\log G(h(n))}{h(n)^{a}} J(n),
$$

where

$$
J(n)=\left\{\frac{1}{a(2 n+1)^{3}}-10 \frac{C_{2}^{\delta(n) \log h(n) / \log C_{1}}}{h(n)^{a \delta(n)}}\right\} .
$$

Now from (6) and (8)-(12) we deduce that

$$
b \delta(n) \log h(n) \geq \log \left(10 a(2 n+1)^{3}\right) .
$$

From this we can easily check that

$$
J(n) \geq 0 .
$$

Since

$$
\begin{aligned}
\log m^{*}(t, f) & =\log |c|+k \log t+\log m^{*}(t, g) \\
& \geq \log |c|+k \log t+\log |G(-t)|
\end{aligned}
$$

and

$$
\begin{aligned}
\log M(t, f) & =\log |c|+k \log t+\log M(t, g) \\
& \leq \log |c|+k \log t+\log G(t),
\end{aligned}
$$

then for $t \geq h(n)$, we have

$$
\begin{aligned}
& \log m^{*}(t, f)-\left[1-\frac{1}{(2 n+1)^{3}}\right] \log M(h(n), f) \\
& \geq \log |G(-t)|-\left[1-\frac{1}{(2 n+1)^{3}}\right] \log G(h(n)) .
\end{aligned}
$$

Combining this with (13)-(15) we have

$$
\int_{h(n)}^{h(n)^{1+\delta(n)}} \frac{\log m^{*}(t, f)-\left[1-1 /(2 n+1)^{3}\right] \log M(h(n), f)}{t^{1+a}} d t \geq 0 .
$$

Thus there exists $t_{n}$ such that (ii) and (iii) hold. 
The following result was proved by Barry [8].

LEMMA 3. Let $f(z)$ be a non-constant entire function with lower order $\rho=\rho(f)<1 / 2$. Then for any $\rho<a<1 / 2$, the lower logarithmic density of the set

$$
E=\left\{r: 0<r<\infty, \log m^{*}(r, f) \leq \cos (\pi a) \log M(r, f)\right\}
$$

satisfies

$$
\underline{\log \text { dens }} E \leq \rho / a \text {. }
$$

Furthermore, if the order $\lambda(f)<1 / 2$, then

$$
\overline{\log \operatorname{dens}} E \leq \lambda / a \text {. }
$$

The following three lemmas are parallel to Baker [1], where he considered the case that the order is less than $1 / 2$.

LEMMA 4. Let $f(z)$ be a non-constant entire function with lower order $\rho=\rho(f)<c<$ $1 / 2$. Then there exists a number $r_{0}>0$ such that, for $r \geq r_{0}$, the interval $\left(r, r^{1+\rho / c}\right)$ contains a value s satisfying

$$
\log m^{*}(s, f) \geq \cos (\pi c) \log M(s, f) .
$$

In addition, for any a with $c<a<1 / 2$, the interval $\left(r, r^{(1+\rho / c) / \cos \pi a}\right)$ contains a value $s$ such that

$$
\log m^{*}(s, f) \geq \log M(r, f)
$$

Proof. For any $0<\varepsilon<1 / 2-c$, we have

$$
\rho<c<c+\varepsilon<1 / 2 .
$$

Note that the lower logarithmic density of the interval $\left(r, r^{1+\rho / c}\right)$ satisfies

$$
\underline{\log \operatorname{dens}}\left(r, r^{1+\rho / c}\right)=\frac{\rho}{c}>\frac{\rho}{c+\varepsilon} .
$$

By Lemma 3, the interval $\left(r, r^{1+\rho / c}\right)$ contains a value $s$ such that

$$
\log m^{*}(s, f)>\cos (\pi(c+\varepsilon)) \log M(s, f) .
$$

Since $s$ is independent of $\varepsilon$, by letting $\varepsilon \rightarrow 0$ we obtain the first conclusion.

Next we prove the second part. For sufficiently large $r$, by the proof of the first conclusion, there exists

$$
s \in\left(r^{1 / \cos \pi a}, r^{(1+\rho / c) / \cos \pi a}\right)
$$

such that

$$
\log m^{*}(s, f)>\cos (\pi c) \log M(s, f) .
$$

Applying the Hadamard-three-circle theorem to the three circles $|z|=1, r, r^{1 / \cos \pi a}$ we get

$$
\log M\left(r^{1 / \cos \pi a}, f\right) \geq \frac{1}{\cos \pi a} \log M(r, f)-\left(\frac{1}{\cos \pi a}-1\right) \log M(1, f) .
$$


Obviously, when $r$ is sufficiently large, we have

$$
\frac{1}{\cos \pi a} \log M(r, f)-\left(\frac{1}{\cos \pi a}-1\right) \log M(1, f) \geq \frac{1}{\cos \pi c} \log M(r, f) .
$$

Note that $\log M(s, f) \geq \log M\left(r^{1 / \cos \pi a}, f\right)$ by the increasing of $\log M(r, f)$, the desired result follows from this and (18)-(20).

LEMMA 5. Let $f(z)$ be a non-constant entire function with lower order $\rho=\rho(f)<1 / 2$. Then for every $a$ and $c$ satisfying $\rho<c<a$, there exists an $R_{a, c}>0$ such that, for $R>R_{a, c}$, the inequalities

$$
M_{-1}(R, f) \leq r(R) \leq\left\{M_{-1}(R, f)\right\}^{L(c, a)}
$$

hold, where $L(c, a)=(1+\rho / c) / \cos (\pi a), r(R)=\inf \left\{r: m^{*}(r, f)=R\right\}$ and $M_{-1}(R, f)$ is the inverse function of $M(R, f)$.

Proof. Let $t=M_{-1}(R, f)$. Then $M(t, f)=R$. On the other hand,

$$
M(r(R), f) \geq m^{*}(r(R), f)=R,
$$

and so, $t \leq r(R)$. This proves the first inequality.

Next we prove the second inequality. Take a number $a^{\prime}$ with $c<a^{\prime}<a$ and define $\delta=\cos \left(\pi a^{\prime}\right) / \cos (\pi a)-1$. Applying Lemma 4 to the interval

$$
I(t)=\left(t^{1+\delta},\left(t^{1+\delta}\right)^{(1+\rho / c) / \cos \pi a^{\prime}}\right)=\left(t^{1+\delta}, t^{L(c, a)}\right),
$$

we see that there exists a value $s \in I(t)$ such that

$$
\log m^{*}(s, f) \geq \log M\left(t^{1+\delta}, f\right) .
$$

On the other hand, for sufficiently large $t$, from the Hadamard-three-circle theorem we deduce that

$$
\begin{aligned}
\log M\left(t^{1+\delta}, f\right) & \geq(1+\delta) \log M(t, f)-\delta \log M(1, f) \\
& \geq \log M(t, f)=\log R
\end{aligned}
$$

This and (22) yield that $m^{*}(s, f) \geq R$. Combining this with the definition of $r(R)$ we get $s \geq r(R)$. Note that $s \in I(t)$ and the conclusion follows.

LEMMA 6. Let $f(z)$ be a non-constant entire function with lower order $\rho=\rho(f)<1 / 2$. Then there is an $R_{0}>0$ such that, for all $R>R_{0}$, the annulus $\left\{z: M_{-1}(R, f) \leq\right.$ $|z|<r(R+1)\}$ contains a simple closed curve $\Gamma$ which contains the origin and on which $|f(z)|=R$.

Proof. (cf. Baker [1, Satz 2]) Let $t=M_{-1}(R, f)$. Then $M(t, f)=R$, and so, $|f(z)|<R$ in $|z|<t$. Meanwhile, from $m^{*}(r(R+1), f)=R+1$ we see that $|f(z)|>R$ on $|z|=r(R+1)$. Thus there exists a component $D$ of the inverse $f^{-1}(|w|<R)$ such that $D$ contains $|z|<t$ and $D$ is contained in $|z| \leq r(R+1)$. Therefore we can find a simple closed curve $\Gamma$ in the annulus $t \leq|z|<r(R+1)$ such that $|f(z)|=R$ on $\Gamma$. In fact, $\Gamma$ is the boundary of $D$, so that it contains the origin. The proof is complete. 
Lemma 7. Suppose that $f(z)$ is a non-constant entire function with lower order $\rho=$ $\rho(f)<1 / 2$. For any $\rho<c<a<1 / 2$, let $L(c, a)$ be the same as in Lemma 5. Then for every positive integer $n$, there exists a constant $r_{0}>0$ such that, for $r>r_{0}$, the ring

$$
r \leq|z|<r^{k(n)} \quad\left(k(n)=L(c, a)^{n}\right)
$$

contains a simple closed curve $\Gamma$ which contains the origin and on which

$$
\left|f^{n}(z)\right|=M\left(r, f^{n}\right) .
$$

Proof. We shall use the method due to Baker [2, Lemma 1]. For sufficiently small $\varepsilon>0$, we take $a^{\prime} \in(c, a)$ such that

$$
(1+\varepsilon) L\left(c, a^{\prime}\right) \leq L(c, a) .
$$

By the Hadamard-three-circle theorem, for any entire function $h(z)$ and any $d>1$, there is an $R_{0}$ such that, for all $R>R_{0}$, we have

$$
M_{-1}\left(R^{d}, h\right)<(1+\varepsilon)\left\{M_{-1}(R, h)\right\}^{d}<\left\{M_{-1}(R, h)\right\}^{d+\varepsilon} .
$$

Thus for small $0<\delta<\varepsilon$, by Lemma 5 ,

$$
\begin{aligned}
r(R+1) & \leq\left\{M_{-1}(R+1, f)\right\}^{L\left(c, a^{\prime}\right)} \\
& \leq\left\{M_{-1}\left(R^{1+\delta}, f\right)\right\}^{L\left(c, a^{\prime}\right)} \\
& \leq\left\{M_{-1}(R, f)\right\}^{(1+\varepsilon) L\left(c, a^{\prime}\right)} \\
& \leq\left\{M_{-1}(R, f)\right\}^{L(c, a)} .
\end{aligned}
$$

Hence, by taking $r_{0}=M_{-1}\left(R_{0}, f\right)$ and $R=M(r, f)$, the conclusion for $n=1$ follows from Lemma 6.

Suppose that the statement is true for a positive integer $m$. We shall prove the truth of the statement for $m+1$, and then by induction, the conclusion follows.

Let $X(c)=1+\rho / c=L(c, a) \cos (\pi a)$. By the first part of Lemma 4, there exists an $R_{0}>0$ such that, for any $R>R_{0}$, there is a value $s$ such that

$$
R<s<R^{X(c)}
$$

and

$$
m^{*}(s, f) \geq M(s, f)^{\cos (\pi c)} .
$$

Now by (23), there exists an $R_{1} \geq R_{0}$ such that, for $R>R_{1}$,

$$
M_{-1}\left(R^{X(c)}, f^{m}\right)<\left\{M_{-1}\left(R, f^{m}\right)\right\}^{X(a)} .
$$

Thus for all sufficiently large $r$ with

$$
r^{1 / \cos (\pi a)}=M_{-1}\left(R, f^{m}\right)
$$

we have

$$
M\left(r^{L(a, a)}, f^{m}\right) \geq R^{X(c)} .
$$


It follows that there exists

$$
t \in\left(r^{1 / \cos (\pi a)}, r^{L(a, a)}\right)
$$

such that

$$
M\left(t, f^{m}\right)=s .
$$

Now by the induction hypothesis there is a simple closed curve $\Gamma_{m}$ in the annulus $t \leq|z|<t^{k(m)}$ which contains the origin and on which

$$
\left|f^{m}(z)\right|=M\left(t, f^{m}\right)=s .
$$

Therefore for $z \in \Gamma_{m}$, from (24) we get

$$
\begin{aligned}
\left|f^{m+1}(z)\right| & \geq m^{*}(s, f) \\
& \geq M(s, f)^{\cos (\pi c)}=\left\{M\left(M\left(t, f^{m}\right), f\right)\right\}^{\cos (\pi c)} \\
& \geq\left\{M\left(t, f^{m+1}\right)\right\}^{\cos (\pi c)} \geq M\left(t^{\cos (\pi a)}, f^{m+1}\right) \\
& \geq M\left(r, f^{m+1}\right) .
\end{aligned}
$$

Let $W(r)=\left\{w:|w|<M\left(r, f^{m+1}\right)\right\}$. Note that $\left|f^{m+1}(z)\right|<M\left(r, f^{m+1}\right)$ in $|z|<r$. Thus there exists a component $D$ of $f^{-(m+1)}(W(r))$ such that $D$ contains $|z|<r$ and $D$ is contained in the interior of $\Gamma_{m}$. Let $\Gamma$ be the boundary of $D$ which is a simple closed curve and is contained in the annulus bounded by $|z|=r$ and $\Gamma_{m}$. Obviously, $\Gamma$ contains the origin and $\left|f^{m+1}(z)\right|=M\left(r, f^{m+1}\right)$ on $\Gamma$. The proof is complete.

LEMMA 8. (Baker [7]) If a transcendental entire function $f$ has a Baker domain D, then

$$
\log \left|f^{n}(z)\right|=O(n) \quad(z \in D, n \rightarrow \infty) .
$$

\section{Proof of Theorem 1}

Define

$$
c(n)=2+\frac{1}{n+1}, \quad n \geq 1 .
$$

For the sequence $R_{n}$ and $t_{n}$ in Lemma 2 we know that

$$
R_{n}<t_{n}<R_{n}^{c(n)}
$$

Since $f$ is transcendental, we may suppose that

$$
\log M\left(R_{n}^{2+2 /(2 n+1)}, f\right)>\frac{4(n+1)(2 n+3)(2 n+1)^{3}}{8 n^{3}+8 n^{2}-2 n-3} \log M(1, f) .
$$

In fact, this holds for all sufficiently large $n$. It follows from the Hadamard-three-circle theorem that

$$
\begin{aligned}
\log R_{n+1} & =\log M\left(R_{n}, f\right) \\
& \leq \log M(1, f)+\frac{1}{2+2 /(2 n+1)} \log M\left(R_{n}^{2+2 /(2 n+1)}, f\right) \\
& \leq \frac{1-1 /(2 n+1)^{3}}{c(n+1)} \log M\left(R_{n}^{2+2 /(2 n+1)}, f\right) .
\end{aligned}
$$

The conclusion follows from Lemma 1. 
4. Proof of Theorem 2

Take any $R_{1}>0$ and define

$$
R_{n+1}=M\left(R_{n}, f\right) \quad n \geq 1 .
$$

For any $a, c$ with $c<a<1 / 2$, let

$$
L(c, a)=(1+\rho / c) / \cos (\pi a), \quad c(n)=L(c, a)^{2} .
$$

By Lemma 4, the interval

$$
\left(R_{n}^{L(c, a)}, R_{n}^{c(n)}\right)
$$

contains a value $t_{n}$ such that

$$
\log m^{*}\left(t_{n}, f\right) \geq \log M\left(R_{n}^{L(c, a)}, f\right) .
$$

Combining this with the hypothesis we get

$$
m^{*}\left(t_{n}, f\right) \geq M\left(R_{n}, f\right)^{c(n)} .
$$

The conclusion follows from Lemma 1 .

\section{Proof of Theorem 3}

Suppose on the contrary that there exists an unbounded pre-periodic component $U$. Then there are two non-negative integers $m$ and $n$ such that $f^{n}(D) \subset D$, where $D=f^{m}(U)$. By Lemma 7 we see that $D$ is unbounded and the only possible constant limit function of $\left\{f^{n j}: j=1,2, \ldots\right\}$ in $D$ is $\infty$, so that $f^{n j}(z) \rightarrow \infty$ in $D$ as $j \rightarrow \infty$. Thus $D$ is a Baker domain of the function $f^{n}$. From Lemma 8 and Lemma 7 we get a contradiction.

Acknowledgements. We wish to thank Professor D. F. Shea for pointing out the topic of lower order to us. We also thank Professor I. N. Baker and the referee for some valuable suggestions.

\section{REFERENCES}

[1] I. N. Baker. Zusammensetzungen ganzer Funktionen. Math. Z. 69 (1958), 121-163.

[2] I. N. Baker. Fixpoints and iterates of entire functions. Math. Z. 71 (1959), 146-153.

[3] I. N. Baker. The domains of normality of an entire function. Ann. Acad. Sci. Fenn. Ser. A I Math. 1 (1975), 277-283.

[4] I. N. Baker. An entire function which has wandering domains. J. Austral. Math. Soc. Ser A. 22 (1976), 173-176.

[5] I. N. Baker. The iteration of polynomials and transcendental entire functions. J. Austral. Math. Soc. Ser. A 30 (1981), 483-495.

[6] I. N. Baker. Wandering domains in the iteration of entire functions. Proc. London Math. Soc. 49 (1984), 563-576.

[7] I. N. Baker. Infinite limits in the iteration of entire functions. Ergod. Th. \& Dynam. Sys. 8 (1988), 503507.

[8] P. D. Barry. On a theorem of Kjellberg. Quart. J. Math. Oxford 15 (1964), 179-191. 
[9] W. Bergweiler and St. Rohde. Omitted values in domains of normality. Proc. Amer. Math. Soc. 123 (1995), 1857-1858.

[10] P. Bhattacharyya. On the domain of normality of an attractive fixpoint. Trans. Amer. Math. Soc. 153 (1971), 89-98.

[11] R. P. Boas. Entire Functions. Academic Press, New York, 1954.

[12] A. Denjoy. Sur un theoreme de Wiman. C. R. Acad. Sci. Paris 193 (1931), 828-830.

[13] R. L. Devaney and F. Tangerman. Dynamics of entire functions near the essential singularity. Ergod. Th. \& Dynam. Sys. 6 (1986), 489-503.

[14] A. E. Eremenko and M. Yu. Lyubich. Iterates of entire functions. Preprint No. 6-84, Fiz.-Tekhn. Inst. Nizkikh Temperatur Akad. Nauk Ukr. SSR. Khar'kov, 1984.

[15] P. Fatou. Sur les équations fonctionelles. Bull. Soc. Math. France 47 (1919), 161-271; 48 (1920), 33-94 and 208-314.

[16] P. Fatou. Sur l' itération des fonctions transcendentes entières. Acta Math. 47 (1926), 337-370.

[17] X. H. Hua and C. C. Yang. Fatou components and a problem of Bergweiler. Int. J. Bifurc. Chaos 8 (1998), 1613-1616.

[18] G. Julia. Mémoire sur l' iteration des fractions rationnelles. J. Math. Pures Appl. 8(1) (1918), 47-245.

[19] B. Kjellberg. On the minimum modulus of entire functions of lower order less than one. Math. Scand. 6 (1960), 189-197.

[20] J. Y. Qiao. Stable domains in the iteration of entire functions. Acta Math. Sinica 37 (1994), $702-708$ (in Chinese).

[21] G. M. Stallard. The iteration of entire functions of small growth. Math. Proc. Camb. Phil. Soc. 114 (1993), 43-55.

[22] D. Sullivan. Quasiconformal homeomorphisms and dynamics I: Solution of the Fatou-Julia problem on wandering domains. Ann. Math. 122 (1985), 402-418. 